\title{
Simulation of magnetostrictive properties of Galfenol under thermomechanical deformation
}

\author{
Abhishek Kumar • Veera Sundararaghavan
}

the date of receipt and acceptance should be inserted later

\section{Abstract}

A recently discovered alloy, Galfenol, has been shown to exhibit magnetostrictive strains up to $400 \mu$ in single crystal form (more than 10 times that of $\alpha-\mathrm{Fe}$ ). However, it has proved difficult to retain the property in the polycrystalline form, for example, cold rolled specimens often lose their magnetostriction. We have developed a microstructure evolution model that is used to study the interplay of microstructure, crystallographic texture development and residual stresses in the evolution of magnetostrictive properties. Finite strain homogenization within a crystal plasticity model is used to investigate microstructural response of processed specimens under coupled magnetic and stress fields.

Keywords: Plastic deformation; Texture; Finite element analysis; Simulation; Theory

\section{Introduction}

When a magnetic field is applied to Galfenol single crystal, the boundaries between the magnetic domains shift and rotate, both of which cause a change in the material's dimensions. This behavior, termed magnetostriction, has been successfully to transduce magnetic field to mechanical force in micro-scale (MEMS) sensors and actuators. While single crystals of Galfenol provide large magnetostriction, their preparation is expensive. It is well known that thermomechanical processes (such as rolling and extrusion) may provide means

A. Kumar · V. Sundararaghavan

Dept. of Aerospace Engineering,

University of Michigan, Ann Arbor, MI-48109, USA

E-mail: veeras@umich.edu to develop polycrystalline textured Galfenol with properties comparable to expensive single crystals $[7 ; 8 ; 9]$. However, it has proved difficult to predict (and thus, control) the large changes in properties such as magnetostriction and yield strength that occur during thermomechanical processing. For example, warm rolled and annealed specimens retain high magnetostriction but are quite brittle; whereas, cold rolled specimens have high yield strength but lose their magnetostriction [5; $6]$. Experimental studies suggest that internal inhomogeneous strains introduced by microstructural changes play an important role in determining the final magnetostriction in Galfenol [11]. To model these aspects, we employ crystal plasticity theory to study the effect of meso-scale features (such as texture, misorientation distribution) on the magnetostrictive response of these alloys. A rate-independent elasto-plastic model of BCC Galfenol single crystal is employed for studying the effect of forming processes on the microstructure response. The properties of the deformed specimens were modeled based on a magnetic free energy description that represents the amount of energy required to rotate a unit volume with a known magnetization to a given direction from a reference direction. The approach was able to well reproduce the experimentally measured magnetostrictive response of cold-worked microstructures in the presence of a variety of external stress states and magnetic field directions.

\section{Computation of magnetostrictive properties}

When a magnetic field is applied to a Galfenol single crystal, the boundaries between the magnetic domains shift and rotate, both of which cause a change in the material's dimensions. Galfenol crystal has minimal en- 
ergy in the $<111>$ family of directions (easy direction of magnetization) and maximal magnetocrystalline energies in the $\langle 100\rangle$ family (hard directions). Magnetostrictive strain is specified using two independent parameters, $\lambda_{100}$ and $\lambda_{111}$, that characterize the changes in normal strain along the $<111>$ and $<100>$ direction resulting from the rotation of a magnetization state into these directions. The magnetostrictive strain tensor for a crystal with magnetization direction given by the unit vector $\boldsymbol{m}=\left(m_{x}, m_{y}, m_{z}\right)$ (in the crystal coordinate system) is then given by the following expression:

$\boldsymbol{\lambda}=\frac{3}{2}\left[\begin{array}{ccc}\lambda_{100}\left(m_{x}^{2}-\frac{1}{3}\right) & \lambda_{111}\left(m_{x} m_{y}\right) & \lambda_{111}\left(m_{x} m_{z}\right) \\ \lambda_{111}\left(m_{y} m_{x}\right) & \lambda_{100}\left(m_{y}^{2}-\frac{1}{3}\right) & \lambda_{111}\left(m_{y} m_{z}\right) \\ \lambda_{111}\left(m_{z} m_{x}\right) & \lambda_{111}\left(m_{z} m_{y}\right) & \lambda_{100}\left(m_{z}^{2}-\frac{1}{3}\right)\end{array}\right]$

A magnetic free energy is then defined that represents the amount of energy required to rotate a unit volume with a known magnetization to a given direction from a reference direction. We use the model from Armstrong [2] that represents the free energy as a sum of internal and external energy terms. The internal energy represents the energy released as the magnetization vector rotates away from a hard direction towards an easier direction of magnetization. The following form of internal energy is taken:

$$
E_{I}=K_{1}\left(m_{x}^{2} m_{y}^{2}+m_{y}^{2} m_{z}^{2}+m_{x}^{2} m_{z}^{2}\right)
$$

The simple form for $E_{I}$ used here ensures that a domain in the crystal has minimal and maximal energies when oriented, respectively, along the $<111>$ directions (easy direction) and the $<100>$ family (hard directions). Application of an external magnetic field leads to an energy change in energy proportional to the intensity of the magnetic field, $\boldsymbol{H}$, the magnetization of the domain, $\boldsymbol{M}$, and the direction between them. The direction of the applied magnetic field is represented as $\boldsymbol{n}=\left(n_{x}, n_{y}, n_{z}\right)$ in the crystal coordinate system.

$$
E_{H}=-\mu_{0} M H(\boldsymbol{m} \cdot \boldsymbol{n})
$$

The energy contribution (per unit volume) associated with the interaction of externally applied stresses with magnetostrictive strains is given as:

$$
E_{\sigma}=-\boldsymbol{\sigma} \cdot \boldsymbol{\lambda}
$$

In an ideal crystal without defects (at $\mathrm{T}=0 \mathrm{~K}$ ), the domain would align in the direction of minimal energy. However, domain magnetization is expected to follow a Boltzmann-like distribution at higher temperatures due to an increase in entropy. The probability, $P$, that the magnetization direction is equal to $\boldsymbol{m}$ is given as:

$P(\boldsymbol{m}) \propto \exp \left(-\frac{\left(E_{I}+E_{H}+E_{\sigma}\right)}{\Omega}\right)$

The parameter $\Omega$ represents the spread of the magnetization direction from the ideal direction (of minimal energy). The magnetostriction strain tensor is obtained by transforming the strains from crystal to sample basis as $\left(\boldsymbol{\lambda}_{s}=R^{T} \boldsymbol{\lambda} R\right)$ where $R$ is the rotation tensor) and averaging over the probability density of magnetization in the crystal.

$$
\langle\boldsymbol{\lambda}\rangle=\frac{\int P(\boldsymbol{m}) \boldsymbol{\lambda}_{\boldsymbol{s}} d m}{\int P(\boldsymbol{m}) d m}
$$

Similarly, the average value of magnetization $(\boldsymbol{M}=\mathbf{M} \boldsymbol{m})$ can be calculated as

$$
\langle\boldsymbol{M}\rangle=\frac{\int P(\boldsymbol{m}) \boldsymbol{M}_{\boldsymbol{s}} d m}{\int P(\boldsymbol{m}) d m}
$$

where $\boldsymbol{M}_{\boldsymbol{s}}=R^{T} \boldsymbol{M} \boldsymbol{m}$ is the magnetization in the sample basis. The above two integrals are calculated by using a finite element representation of the surface of a unit sphere (with 320 quadrilateral elements). Each point on the unit sphere represents a unit normal vector (magnetization direction). The free energy is computed over all the integration points for each element and the integral over $\boldsymbol{m}$ is computed by summing up the element contributions. The actual magentostrictive strains are calculated by subtracting out the strains for an unstressed reference crystal of same orientation, but with zero applied magnetic field. The computed strains for each integration point in the FE mesh is then averaged in Eq.(6) to compute the overall magnetization strain in the material.

A total Lagrangian FEM formulation is used to solve the microstructural response after unloading from a deformation process, which leads to residual elastic stress $\left(\boldsymbol{\sigma}_{\text {res }}\right)$ that interact with the magnetostrictive strains. The unloading process is modeled as a non-linear (finite deformation) elasto-static boundary value problem [12]. Here, it is assumed that the total strain $(\boldsymbol{\epsilon})$ at any point in the material consists of a linear superposition of elastic strain $\left(\boldsymbol{\epsilon}_{e}\right)$ and magnetostrictive strain. From Hooke's law,

$\boldsymbol{\sigma}=\overline{\boldsymbol{C}} \boldsymbol{\epsilon}_{e}=\overline{\boldsymbol{C}}(\boldsymbol{\epsilon}-\lambda)$

where $\overline{\boldsymbol{C}}$ is the fourth-order cubic elasticity tensor for bcc galfenol.

To solve for the final displacements after magnetic loading of the processed specimen, a Newton Raphson iterative scheme $K \delta u=R$ is employed, where, stiffness 
matrix $(\mathrm{K})$ and residual (R) are calculated in terms of $\overline{\boldsymbol{C}}$ and $\lambda$ as

$$
\begin{aligned}
& R=-\int_{\Omega} B^{T} \bar{C} \epsilon d \Omega+\int_{\Omega} B^{T} \bar{C} \lambda d \Omega+\int_{\Gamma} N^{T} \bar{t} d \Gamma \\
& K=\int_{\Omega} B^{T} \bar{C} B d \Omega-\int_{\Omega} B^{T} \bar{C} \frac{\partial \lambda}{\partial u} d \Omega
\end{aligned}
$$

Standard Galerkin formulation is used to calculate shape functions $(\mathrm{N})$ and derivative of shape functions (B). To calculate $\frac{\partial \lambda}{\partial \sigma}$, the equation is first written as $\frac{\partial \lambda}{\partial u}=\frac{\partial \lambda}{\partial \sigma} \frac{\partial \sigma}{\partial u}$ and a perturbation approach is applied on the model in Eq.(6) to compute $\frac{\partial \lambda}{\partial \sigma}$.

\section{Process model}

A rate-independent single-crystal plasticity model developed in Kothari and Anand [1] is used to compute the effect of macroscopic strain on the polycrystal. For a material with $\alpha=1, \ldots, N$ slip systems defined by ortho-normal vector pairs $\left(\boldsymbol{m}^{\alpha}, \boldsymbol{n}^{\alpha}\right)$ denoting the slip direction and slip plane normal respectively, the constitutive equations relate the following basic fields: the deformation gradient $\boldsymbol{F}$ which can be decomposed into elastic and plastic parts as $\boldsymbol{F}=\boldsymbol{F}^{e} \boldsymbol{F}^{p}$, the Cauchy stress $\boldsymbol{T}\left(=\frac{1}{\operatorname{det} \boldsymbol{F}_{r}} \boldsymbol{P}_{r} \boldsymbol{F}_{r}^{T}\right)$ and the slip resistances $s^{\alpha}>0$. In the constitutive equations, the Green elastic strain measure $\overline{\boldsymbol{E}}^{e}=\frac{1}{2}\left(\boldsymbol{F}^{e T} \boldsymbol{F}^{e}-\boldsymbol{I}\right)$ defined on the relaxed configuration (plastically deformed, unstressed configuration) is utilized. The conjugate stress measure is then defined as $\overline{\boldsymbol{T}}=\operatorname{det} \boldsymbol{F}^{e}\left(\boldsymbol{F}^{e}\right)^{-1} \boldsymbol{T}\left(\boldsymbol{F}^{e}\right)^{-T}$ where $\boldsymbol{T}$ is the Cauchy stress for the crystal in the sample reference frame. The constitutive relation, for stress, is given by $\overline{\boldsymbol{T}}=\overline{\boldsymbol{C}}\left[\overline{\boldsymbol{E}}^{e}\right]$.

It is assumed that deformation takes place through dislocation glide and the evolution of the plastic flow is given by

$\dot{\boldsymbol{F}}^{p}\left(\boldsymbol{F}^{p}\right)^{-1}=\sum_{\alpha} \dot{\gamma}^{\alpha} \boldsymbol{S}_{0}^{\alpha} \operatorname{sign}\left(\tau^{\alpha}\right)$

where $\boldsymbol{S}_{0}^{\alpha}=\boldsymbol{m}^{\alpha} \otimes \boldsymbol{n}^{\alpha}$ is the Schmid tensor and $\dot{\gamma}^{\alpha}$ is the plastic shearing rate on the $\alpha^{t h}$ slip system. The resolved stress on the $\alpha^{\text {th }}$ slip system is given by $\boldsymbol{\tau}^{\alpha}=$ $\overline{\boldsymbol{T}} \cdot \boldsymbol{S}_{0}^{\alpha}$. The resolved shear stress $\boldsymbol{\tau}^{\alpha}$ attains a critical value $s^{\alpha}$ on the systems where slip occurs $\left(\dot{\gamma}^{\alpha}>0\right)$. Further, the resolved shear stress does not exceed $s^{\alpha}$ on the inactive systems with $\dot{\gamma}^{\alpha}=0$. The hardening law for the slip resistance $s^{\alpha}$ is taken as,

$\dot{s}^{\alpha}(t)=\sum_{\beta} h^{\alpha \beta} \dot{\gamma}^{\beta}, s^{\alpha}(0)=s_{0}^{\alpha}$

The hardening moduli is given as:

$h^{\alpha \beta}=\left[q+(1-q) \delta^{\alpha \beta}\right] h_{o}\left(1-\frac{s^{\beta}}{s_{s}}\right)^{a}($ no sum on $\beta)$

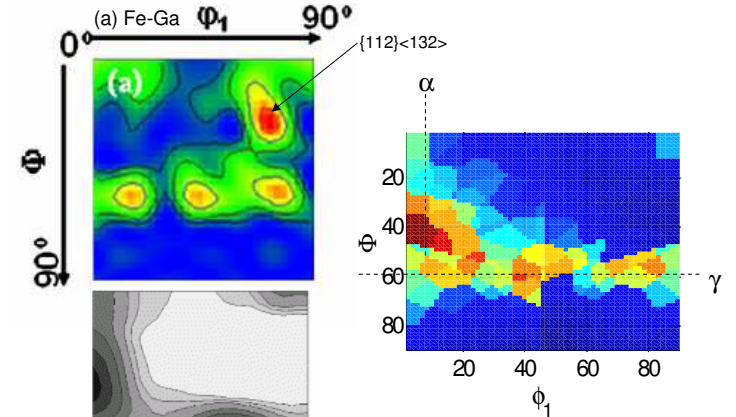

(b) Simulation

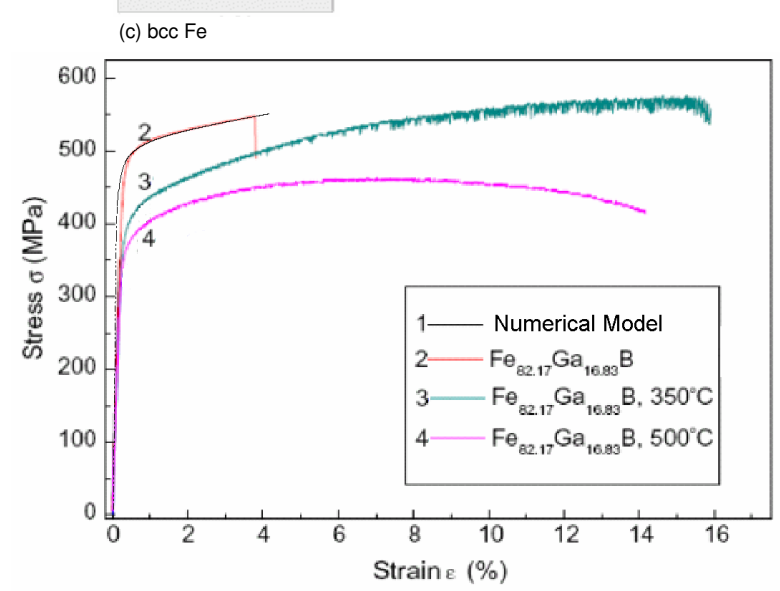

Fig. 1 (left) Comparison of textures (Euler angle space, $\phi_{2}=45^{\circ}$ ) predicted by our model (Fig. 1(b)) with experiments on BCC iron in Fig. 1(c) [4]. Experimental rolling textures of BCC Fe-16.83\% Ga results (Fig 1(a) [10]) are also shown. The experiment indicates a $\{112\}<132>$ texture in addition to the expected $\gamma$ texture. (right) Comparison of results of current model with published results in [10]. The plot shows tensile test curves of as-cast polycrystalline Galfenol at different temperatures.

where $h^{\beta}$ is a single slip hardening rate, $q$ is the latenthardening ratio and $\delta^{\alpha \beta}$ is the Kronecker delta function.

\section{Results}

In the examples, the parameter $q$ is taken to be 1.0 for coplanar slip systems and 1.4 for non-coplanar slip systems. Here $h_{o}, a$, and $s_{s}$ are slip hardening parameters taken to be identical for all slip systems, with values $h_{o}=500 \mathrm{MPa}, s_{s}=350 \mathrm{MPa}$ and $a=2.25$ for BCC Galfenol single crystals. The initial value of slip system resistance is calibrated as $s_{o}=180 M P a$. Values of elastic parameters for Galfenol crystal are taken as $C_{11}=$ $213 \mathrm{GPa}, C_{12}=174 \mathrm{GPa}$ and $C_{44}=120 \mathrm{GPa}$. The initial texturing of the material is assumed to be random. Plastic deformation due to crystallographic slip is assumed to occur in the $\langle 111>$ direction, and the possible slip planes are of the $\{110\},\{112\}$, and $\{123\}$ 
type. The model adequately captures the macroscopic tensile mode stress-strain response at room temperature reported in [10] well as shown in Fig. 1(right). To further validate the microscale model, we compared the results with textures seen in BCC iron rolling processes and textures predicted by our model. The model results from Fig. 1(b) captures both $\alpha$ and $\gamma$ texture seen from experiments (in Fig. 1(c) [4]). Results were also compared with experimental rolling textures of BCC Fe-16.83\% Ga results (Fig 1(a) [10]).

The magnetostrictive model developed here was subsequently validated by comparing with results in literature [3]. The values of constants used in this study are as follows:

$K_{1}=3.6 \times 10^{4} \mathrm{~J} / \mathrm{m}^{-1}$

$(3 / 2) \lambda_{100}=255 \times 10^{-6} \quad(3 / 2) \lambda_{111}=-7 \times 10^{-6}$

$M_{s}=1.83 / \mu_{0} \mathrm{Am} m^{-1} \quad \Omega=625 \mathrm{~J} / \mathrm{m}^{-3}$

Our model includes two enhancements to a single crystal model to better account for the behavior of polycrystalline Galfenol. Firstly, we use a 3D model that includes all stress components in the crystal (this includes the effect of shear stress produced by rolling or any other mechanical processes). Secondly, instead of volume averaging, we have employed a finite element homogenization approach to compute the effective properties.

We show the $\lambda-\mathrm{H}$ and B-H plot of $18 \%$ Ga singlecrystal FeGa in the [100], [210] and [111] directions for various compressive pre-stress values $(0,5,20,40$ and 80 $\mathrm{MPa})$ in Fig. 2. In [100] case, the stress and magnetic field is applied along [100] crystallographic direction. In the plots, the continuous line (3D model) is from this work and dotted line (1D model) is from Ref. [3]. The plots for $\lambda-\mathrm{H}$ show good comparison with deviations at the highest compressive stresses but the saturation value is same for both models. Once we got the good comparison for single-crystal case, we used our model to obtain experimental actuation behavior for polycrystals which is shown in Fig.5(left). These plots are also in good agreement with published results [3] in Fig.5(right).

The effect of a rolling process on polycrystalline Galfenol was subsequently studied. A microstructure with 31 grains was generated using a standard Voronoi tessellation based on our previous work [12]. Texture was randomly assigned and the microstructure was discretized into 690 quadrilateral elements. A rolling process (with plane strain compression along y-axis) was studied with a strain rate of $10^{-3}$ for a time of $10 \mathrm{sec}-$ onds. The microstructure was subsequently unloaded to study the effect of the rolling process. After unloading from a strain of $1 \%$, a spring back of $0.065 \%$ was observed in the y-direction. Using the magnetostriction model, the final magnetostrictive state was computed over each element. Here, a $20000 \mathrm{~A} /$ turn (=251.33 Oe) magnetic field was applied along the y-direction. The magnetostrictive strains along the $\mathrm{x}$ - and $\mathrm{y}$ - directions, respectively, are plotted in Fig. 5. It is seen that grains with high $\mathrm{x}$ - strains are associated with low y- strains and vice versa. Significant changes in magnetostriction strains are seen even within a single grain due to the effect of misorientations and residual stresses.

Finally, we studied the effect of annealing on magnetostrictive behavior. In Fig. 4, three cases are plotted. Case 1 is Galfenol without any processing, case 2 involves rolling Galfenol for 10 s and case 3 involves rolling Galfenol for 10 s and then annealing. Cold working is known to deteriorate the magnetostrictive properties by inducing residual stresses that couple with the subsequently applied magnetic fields. As we see from the plot, by removing the residual stresses, an annealing process is able to partially restore the magnetostrictive behavior.

\section{Conclusion}

We have developed a rate-independent elasto-plastic model of BCC Galfenol single crystal for studying the effect of microstructure on mechanical properties. Both loading and unloading processes have been simulated and a finite strain homogenization algorithm has been developed to investigate final microstructural response. The 3D model developed reproduce experimental behavior of polycrystalline Galfenol under coupled magnetic and stress fields. This study shows that internal inhomogeneous strains introduced by microstructural changes play an important role in determining the final magnetostriction in Galfenol. Significant changes in magnetostriction strains are seen even within a single grain due to the effect of misorientations and residual stresses. Cold work creates residual stresses that reduce magnetostriction in polycrystalline galfenol. However, removal of these residual stresses through annealing significantly improves the magnetostrictive strains. Microstructure-sensitive design methods can then employ the model developed in this work to address inverse/optimization problems such as computation of optimal crystal orientation distributions that lead to desired elasto-plastic properties [13] and identification of processing paths that lead to optimal microstructures [14]. Such new processing routes can be used to produce polycrystalline galfenol with improved magnetostrictive strains for a variety of sensing applications. 


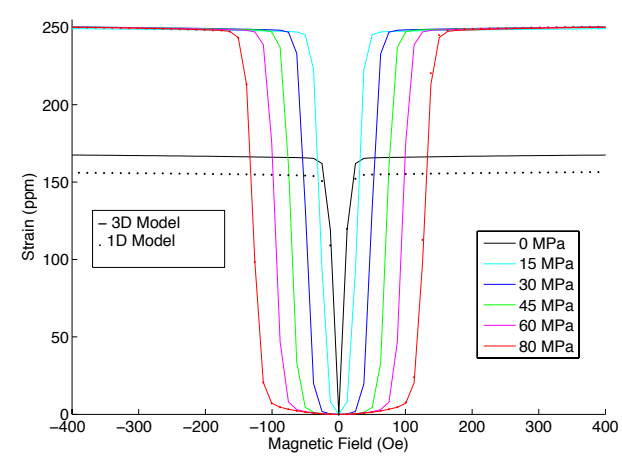

(a) $\lambda H_{100}$

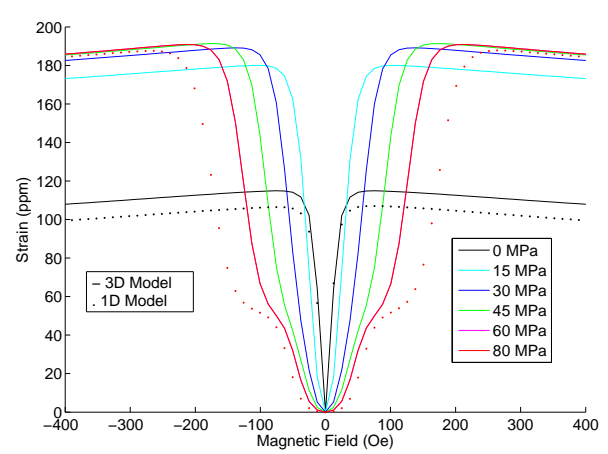

(c) $\lambda H_{210}$

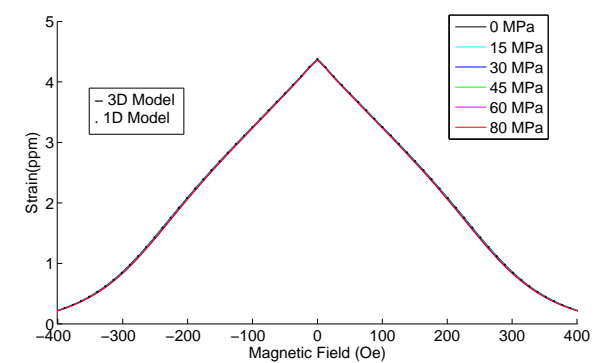

(e) $\lambda H_{111}$

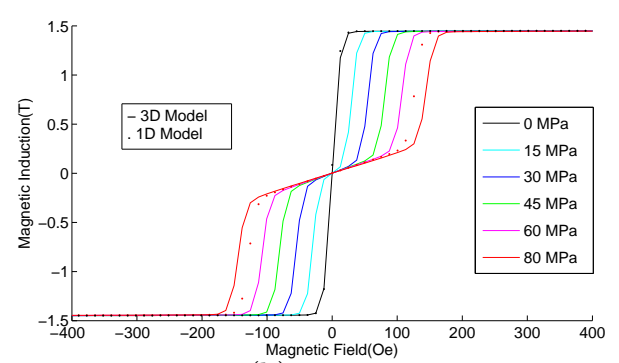

(b) $B H_{100}$

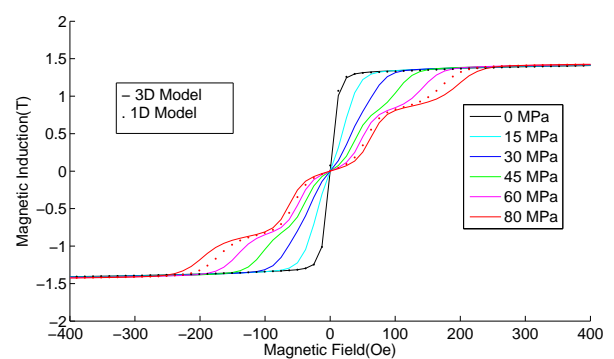

(d) $\mathrm{BH}_{210}$

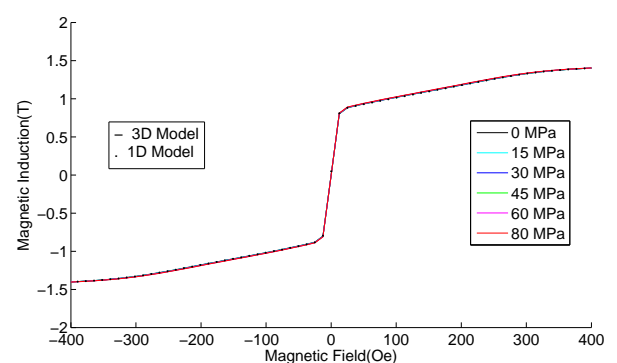

(f) $B H_{111}$

Fig. 2 Magnetostrictive $\lambda-H$ and B-H response for various compressive pre-stress values of $0,15,30,45,60$ and $80 \mathrm{MPa}$ along [100], [210] and [111]crystallographic direction.

\section{Acknowledgements}

This work was supported by the Air Force Office of Scientific Research (AFOSR), Department of Defense (DOD) Award No. FA9550-12-1-0458 and National Science Foundation Award No. 0954390.

\section{References}

1. L. Anand and M. Kothari. A computational procedure for rate-independent crystal plasticity,. Jour- nal of the Mechanics and Physics of Solids, 44:525558, 1996.

2. W. D. Armstrong. Nonlinear behavior of magnetostrictive particle actuated composite materials,Journal of applied physics, 87(6):3027-3031, 2000.

3. J. Atulasimha, A. B. Flatau, and E. Summers. Characterization and energy-based model of the magnetomechanical behavior of polycrystalline iron gallium alloys. Smart Mater Struct, 16:12651276,2007 . 

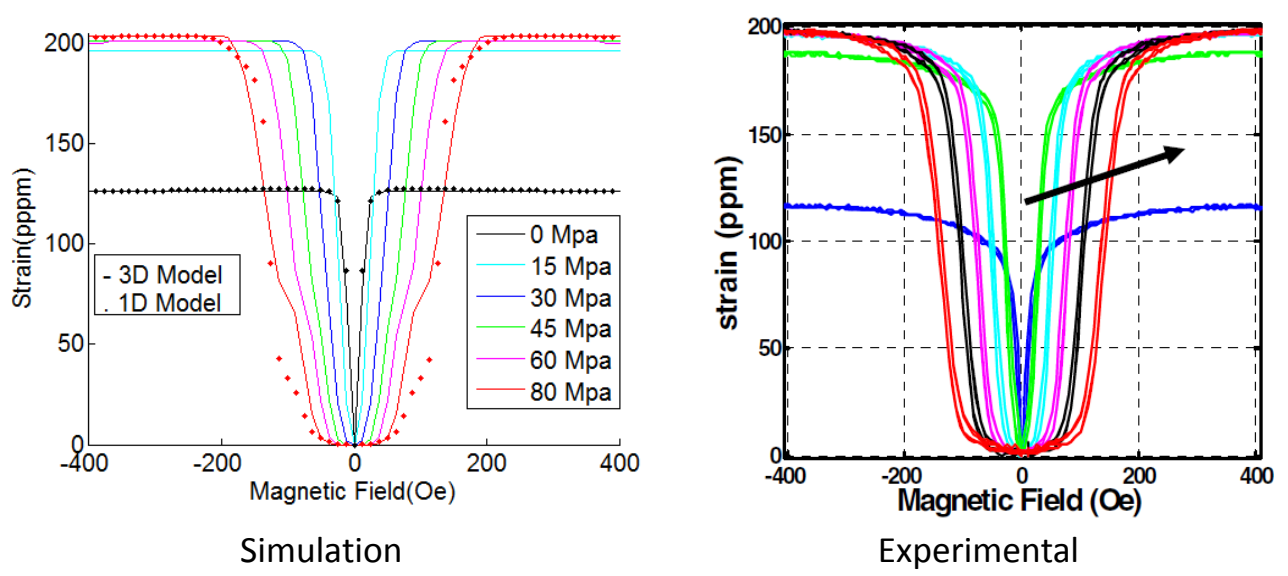

Fig. 3 (left) Computed magnetostrictive $\lambda-H$ response of polycrystal for various compressive pre-stress values of $0,15,30$, 45, 60 and $80 \mathrm{MPa}$. Experimental results from [3] is shown on the right for comparison.

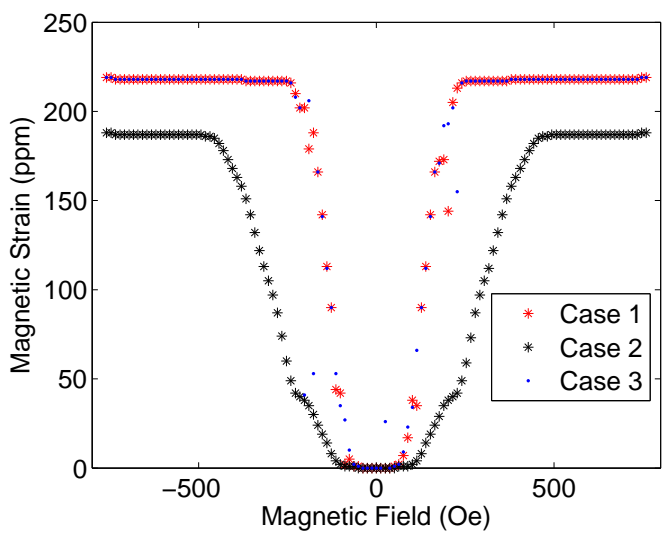

Fig. 4 Effect of Annealing Process on magnetostriction process. Case 1 is Galfenol without any processing, case 2 involves rolling Galfenol for 10 s and case 3 involves rolling Galfenol for 10 s and then annealing.

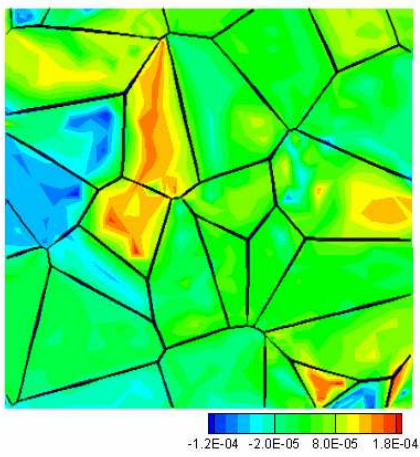

(a)

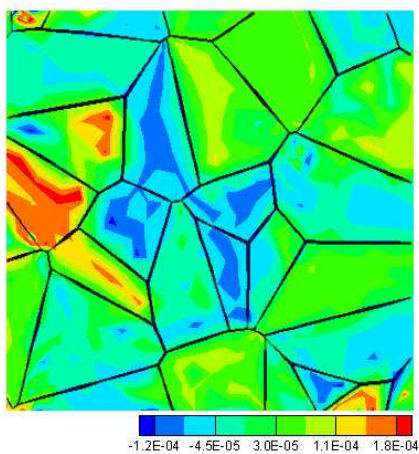

(b)
Fig. 5 Magnetostrictive strain distribution in the as-rolled microstructure (to $1 \%$ strain) under a y-direction magnetic field of 251.33 Oe. (a) Magnetostrictive strains along $\mathrm{x}$-direction and (b) strains along the $\mathrm{y}$ - direction.
4. P. S. Bate and J. Quinta da Fonseca. Texture development in the cold rolling of if steel. Materials Science and Engineering A, 380(1-2):365377,2004 .

5. L. M. Cheng, A. E. Nolting, B. Voyzelle, and C. Galvani. Deformation behavior of polycrystalline galfenol at elevated temperatures. In Marcelo Dapino, editor, Behavior and Mechanics of Multifunctional and Composite Materials, page pp. 65262 N. J Proceedings of the SPIE, Volume 6526 , 2007.

6. Na, S.M. and Flatau, A.B., 2008. Deformation behavior and magnetostriction of polycrystalline fegax $(\mathrm{x}=\mathrm{b}, \mathrm{c}, \mathrm{mn}, \mathrm{mo}, \mathrm{nb}, \mathrm{nbc})$ alloys. Journal of Applied Physics, 103(7), p.07D304.

7. R. A. Kellogg, A. M. Russell, T. A. Lograsso, A. B. Flatau, A. E. Clark, and M. Wun-Fogle. Tensile properties of magnetostrictive iron gallium alloys. Acta Mater. 52 (2004), pages 5043-5050.

8. J. B. Restorff, M.WunFogle, and A. E. Clark, Measurement of d15 in Fe100-xGax $(\mathrm{x}=12.5 ; 15 ; 18.4 ; 22), \quad \mathrm{Fe} 50 \mathrm{Co} 50$, and Fe81Al19 highly textured polycrystalline rods, J. Appl. Phys. 103 (2008) pp. 07B305.

9. Mahadevan, A., P. G. Evans, and M. J. Dapino. Dependence of magnetic susceptibility on stress in textured polycrystalline Fe81.6Ga18.4 and Fe79.1Ga20.9 Galfenol alloys. Applied Physics Letters 96(1) (2010) p.2502.

10. J. H. Li, X. X. Gao, J. Zhu, X. Q. Bao, T. Xia, and M. C. Zhang. Ductility, texture and large magnetostriction of fe-Ga-based sheets. Scripta Materialia, 63(246-249), 2010.

11. N. Srisukhumbowornchai and S. Guruswamy. Crystallographic textures in cold-rolled and annealed fe-ga and fe-al alloys, metallurgical materi- 
als transactions a, 35a (2004). Metallurgical Materials Transactions A, 35A (2004), pages 29632970.

12. V. Sundararaghavan and N. Zabaras, Design of microstructure-sensitive properties in elastoviscoplastic polycrystals using multi-scale homogenization, International Journal of Plasticity, Vol. 22, pp. 1799-1824, 2006.

13. R. Liu, A. Kumar, Z. Chen, A. Agrawal, V. Sundararaghavan and A. Choudhary, A predictive machine learning approach for microstructure optimization and materials design, Nature Scientific Reports, 5(11551),2015.

14. V. Sundararaghavan and N. Zabaras, Linear analysis of texture-property relationships using process-based representations of Rodrigues space, Acta Materialia, Vol. 55, Issue 5, pp. 1573-1587, 2007. 